\title{
High-resolution spectroscopy of globular cluster post-Asymptotic Giant Branch stars ${ }^{\star}$
}

\author{
C. J. Mooney ${ }^{1,2}$, W. R. J. Rolleston ${ }^{1}$, F. P. Keenan ${ }^{1}$, P. L. Dufton ${ }^{1}$, J. V. Smoker ${ }^{1,4}$, \\ R. S. I. Ryans ${ }^{1}$, L. H. Aller ${ }^{3}$, and C. Trundle ${ }^{1,2}$
}

1 Department of Pure \& Applied Physics, The Queen's University of Belfast, Belfast, BT7 1NN, Northern Ireland, UK
2 Isaac Newton Group, Apartado de Correos 368, Santa Cruz de La Palma, Tenerife 38780, Canary Islands, Spain
3 Physics and Astronomy Department, University of California, Los Angeles, CA 90095, USA
${ }^{4}$ European Southern Observatory, Alonso de Cordova 3107, Casilla 19001, Vitacura, Santiago 19, Chile

Received 5 April 2002 / Accepted 12 February 2004

\begin{abstract}
We present model atmosphere analyses of high resolution Keck and VLT optical spectra for three evolved stars in globular clusters, viz. ZNG-1 in M 10, ZNG-1 in M 15 and ZNG-1 in NGC 6712. The derived atmospheric parameters and chemical compositions confirm the programme stars to be in the post-Asymptotic Giant Branch (post-AGB) evolutionary phase. Differential abundance analyses reveal CNO abundance patterns in M 10 ZNG-1, and possibly M 15 ZNG-1, which suggest that both objects may have evolved off the AGB before the third dredge-up occurred. The abundance pattern of these stars is similar to the third class of optically, bright post-AGB objects discussed by van Winckel (1997). Furthermore, M 10 ZNG-1 exhibits a large $\mathrm{C}$ underabundance (with $\Delta[\mathrm{C} / \mathrm{O}] \sim-1.6 \mathrm{dex}$ ), typical of other hot post-AGB objects. Differential $\Delta[\alpha / \mathrm{Fe}]$ abundance ratios in both M 10 ZNG-1 and NGC 6712 ZNG-1 are found to be approximately 0.0 dex, with the Fe abundance of the former being in disagreement with the cluster metallicity of M10. Given that the Fe absorption features in both M 10 ZNG-1 and NGC 6712 ZNG-1 are well observed and reliably modelled, we believe that these differential Fe abundance estimates to be secure. However, our Fe abundance is difficult to explain in terms of previous evolutionary processes that occur on both the Horizontal Branch and the AGB.
\end{abstract}

Key words. Galaxy: globular clusters: individual: M 10, M 15, NGC 6712 - stars: early-type - stars: AGB and post-AGB stars: abundances

\section{Introduction}

Planetary nebulae (PNe) are believed to originate from low to intermediate mass stars $\left(\sim 1-8 M_{\odot}\right)$, which undergo extensive mass-loss during their Asymptotic Giant Branch (AGB) phase of evolution (see the review by Aller 1994). Stellar evolution from the AGB and beyond is still relatively poorly understood but the chemical compositions of the atmospheres and ejecta of PNe stars should provide information on nucleogenesis processes in the stellar interiors, and on mechanisms whereby products are transported to the stellar photosphere. Observed abundances of $\mathrm{He}, \mathrm{N}$ and to a lesser extent $\mathrm{C}, \mathrm{O}$ normally carry the imprint of nuclear processing, whereas abundances of heavier elements should convey the state of the progenitor interstellar medium.

To date, abundances have mainly been estimated from the nebular emission from PNe ejecta (see Aller et al. 1999 and

Send offprint requests to: F. P. Keenan,

e-mail: f.keenan@qub.ac.uk

* Equivalent width data table is only available in electronic form at the CDS via anonymous ftp to cdsarc.u-strasbg.fr $(130.79 .128 .5)$ or via

http://cdsweb.u-strasbg.fr/cgi-bin/qcat?J/A+A/419/1123 references therein). However, such estimates may not provide reliable chemical compositions since: (i) much of the ejected matter is locked up in grains (especially the refractory elements $\mathrm{Si}, \mathrm{Fe}$ etc.) and is not detectable via emission line spectroscopy, and (ii) there will be unobserved ionization stages in any nebular spectrum which may lead to underestimates of the total element abundances (Aller 1990).

A more reliable method for determining element abundances during the later stages of stellar evolution is provided by post-Asymptotic Giant Branch (post-AGB) stars. Post-AGB (or proto-PNe) stars are objects which have recently evolved off the AGB but have not reached a high enough effective temperature to fully ionise a PNe. This evolutionary stage is very short lived (typically $10^{3}$ to $10^{5}$ years) during which time no additional chemical evolution should occur (Schönberner \& Blöcker 1996; Mowlavi 1999). As a result, the derived abundances of post-AGB stars will provide accurate estimates of $\mathrm{PNe}$ chemical compositions and improve our understanding of the physical and chemical changes that occur during the late AGB phase.

In particular, B-type post-AGB stars with effective temperatures $\left(T_{\text {eff }}\right)$ in the range $\sim 10000-30000 \mathrm{~K}$ show optical spectra rich in absorption lines, suitable for measuring the 
abundances of several elements (e.g. He, C, N, O, Mg, Si, Fe). As a result of a continuing programme to investigate B-type stars in the halo of our Galaxy (Keenan 1992), we have identified several hot post-AGB stars on the basis of their derived atmospheric parameters and chemical compositions (Conlon et al. 1991, 1993, 1994; McCausland et al. 1992; Mooney et al. 2001). Although these hotter stars should be evolving from their cooler counterparts, the abundance patterns of the former appear to display significant differences to those of the latter, most notably for carbon, with significant underabundances having been observed in several hot post-AGB stars (see, for example, Conlon et al. 1991, 1994; Mooney et al. 2001). Indeed, the observed CNO abundance pattern in the hot post-AGBs appears to be incompatible with the products of helium burning which should be mixed to the stellar surface via the third dredge-up on the AGB. Thus, it has been suggested that these hot post-AGB candidates appear to have left the AGB before undergoing the third dredge-up.

The current discrepancies between the chemical compostions found for hot and cool post-AGB objects requires further investigation. We have therefore initiated a high resolution spectroscopic programme of known hot post-AGB objects, viz. the UV-bright stars found in some globular clusters. Here the results of model atmosphere analyses of three such globular cluster post-AGB stars, (M 10 ZNG-1, M 15 ZNG-1 and NGC 6712 ZNG-1) are reported. The principle aims of this investigation are to understand the evolutionary status of these objects and to provide templates which will allow the accurate identification of field post-AGB stars.

\section{Observations and data reduction}

NGC 6712 ZNG-1 was observed on the 4 July 2000 with the 10-m Keck I telescope using the HIRES échelle spectrometer (HIRESb) and 53 grooves $\mathrm{mm}^{-1}$ grating + Tektronix $2 \mathrm{~K} \times 2 \mathrm{~K}$ CCD. Complete wavelength coverage from approximately 3650 to $5170 \AA$ was obtained at a spectral resolution of $R \sim 60000$ and a signal-to-noise $(S / N)$ ratio of approximately 60 . M 10 ZNG-1 and M 15 ZNG-1 were observed during the period 01-03 July 2001 with the 8-m VLT KUEYEN telescope, using the Ultraviolet Visible échelle spectrometer (UVES) and 41.6 grooves $\mathrm{mm}^{-1}$ grating $+2 \mathrm{~K} \times 4 \mathrm{~K}$ EEV CCD. Complete wavelength coverage from 3760 to $4980 \AA$ was obtained with $R \sim 40000$ and $S / N$ ratios of 170 and 60 respectively.

Balmer hydrogen line profiles were centered in their échelle orders (allowing reliable surface gravity estimates), whilst also permitting the observation of neutral He and important metallic features (to characterise chemical composition). Although such metal lines may be weak in post-AGB stars, the Keck and VLT instruments are ideal for achieving the high spectral resolution and $S / N$ data required. The stellar observations were of 20-30 min duration, and bracketed by Th-Ar lamp exposures for wavelength calibration. Flat-field exposures, made using quartz continuum lamps, were taken at the beginning of the night, while bias frames were acquired at the end.

Reduction of the raw 2-dimensional images to onedimensional spectra was undertaken using standard procedures within the MAuna Kea Echelle Extraction (MAKEE) data reduction package for the Keck data, and the UVES Data Reduction Pipeline package for the VLT data. Some examples of the reduced and normalised spectra are illustrated in Fig. 1. After extraction, the spectra were analysed using the Starlink package DIPSO (Howarth et al. 1994).

For reasons discussed in Sect. 5, we believe that a differential abundance analysis may provide a more accurate picture of the chemical composition of post-AGB stars. Hence, the postAGBs were analysed with respect to standard stars with normal Population I chemical compositions (Gies \& Lambert 1992). M 10 ZNG-1 and M 15 ZNG-1 were analysed with respect to the Galactic disc star HR 2387 ( $\equiv \xi^{1} \mathrm{CMa}$ ), which was observed during runs at the 0.9-m Coudé Feed telescope at Kitt Peak National Observatory (KPNO) and the 2.7-m telescope of the University of Texas McDonald Observatory (see Hambly et al. 1996 for further details). NGC 6712 ZNG-1 was analysed with respect to HR 6825 and $\beta$ Ori; both observed with UCLES instrument at the Anglo-Australian Telescope and reduced using standard methods (see, for example Mooney et al. 2001).

\section{Method of analysis}

\subsection{Radial velocity and equivalent width measurement}

Stellar radial velocities were estimated from Doppler shifts of strong isolated metal lines in the spectra of the programme stars. This resulted in mean Local Standard of Rest radial velocities of $\Delta v_{\mathrm{lsr}}=+91.7 \mathrm{~km} \mathrm{~s}^{-1}, \Delta v_{\mathrm{lsr}}=-99.6 \mathrm{~km} \mathrm{~s}^{-1}$, $\Delta v_{\mathrm{lsr}}=-100.0 \mathrm{~km} \mathrm{~s}^{-1}$ for M $10 \mathrm{ZNG}-1$, M $15 \mathrm{ZNG}-1$ and NGC 6712 ZNG-1 respectively. The equivalent widths of all unblended metal lines were estimated by employing nonlinear least-squares Gaussian fitting routines in DIPSO, with line centre, width and strength as variable parameters. Continuum distributions were estimated by fitting low order $(\leq 4)$ leastsquares polynomials in the regions of spectra free from absorption features. The quality of the observational data allowed equivalent widths down to approximately $10 \mathrm{~m} \AA$ to be reliably measured with values as small as $4-5 \mathrm{~m} \AA$ being estimated for M 10 ZNG-1, which has the highest $S / N$ ratio spectroscopy. The equivalent width data are too extensive to be listed here, but are available at the CDS archive.

For the hydrogen and diffuse helium lines, normalised profiles were directly extracted. The échelle orders containing these strong and broad lines were difficult to normalize, particularly given the significant échelle ripple. In such cases, the continuum fits of adjacent orders were interpolated, as discussed by McCausland et al. (1992) and used to rectify the relevant order prior to normalisation. An example of a reduced spectrum for a broad hydrogen line is shown in Fig. 1.

\subsection{Stellar atmospheric parameters}

Observed features in the spectra of M 10 ZNG-1, M 15 ZNG-1 and NGC 6712 ZNG-1 have been compared with model atmospheres generated using TLUSTY (see Hubeny 1988; Hubeny \& Lanz 1992, 1995) and LTE radiative transfer codes (see, for example, Mooney et al. 2001). 
Table 1. Adopted atmospheric and fundamental parameters of the programme stars.

\begin{tabular}{lccccccc}
\hline \hline Star & $\alpha_{\text {J2000 }}$ & $\delta_{\text {J2000 }}$ & $\begin{array}{c}T_{\text {eff }} \\
(\mathrm{K})\end{array}$ & $\begin{array}{c}\log g \\
(\mathrm{dex})\end{array}$ & $\begin{array}{c}\xi \\
\left(\mathrm{km} \mathrm{s}^{-1}\right)\end{array}$ & $V_{[\mathrm{mag}]}^{1}$ & $(B-V)^{1}$ \\
\hline M 10 ZNG-1 & 165709.4 & -040423.8 & $27000 \pm 1000$ & $3.6 \pm 0.2$ & 15 & 13.23 & -0.30 \\
M 15 ZNG-1 & 212958.1 & +121144.0 & $28000 \pm 1000$ & $3.7 \pm 0.2$ & 10 & 14.80 & -0.32 \\
NGC 6712 ZNG-1 & 185305.7 & -084239.4 & $11000 \pm 1000$ & $2.1 \pm 0.2$ & 10 & 13.00 & -0.06 \\
\hline
\end{tabular}

${ }^{1}$ de Boer 1987.
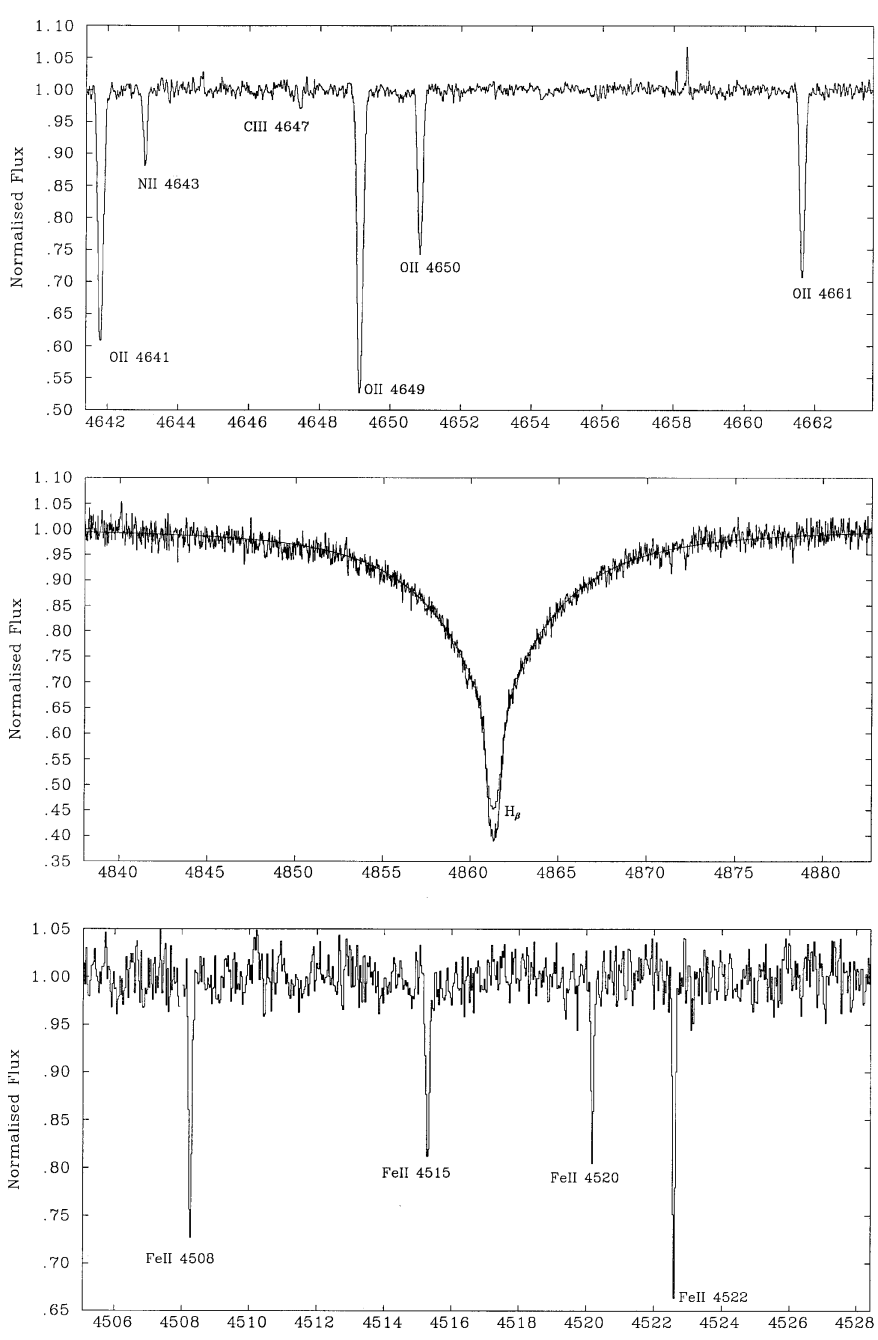

Fig. 1. The normalised spectra of M10 ZNG-1, M 15 ZNG-1 and NGC 6712 ZNG-1. Top panel: M10 ZNG-1 over the spectral range 4642-4662 A, illustrating $\mathrm{C}, \mathrm{N}$ and $\mathrm{O}$ absorption features. Middle panel: the normalised Balmer $\mathrm{H}_{\beta}$ line in M 15 ZNG-1. The theoretical $\mathrm{H}_{\beta}$ profile is shown as a solid line. Bottom panel: Fe II lines in NGC 6712 ZNG-1 over the spectral range $\sim 4505-4528 \AA$.

\subsection{1. $M 10 Z$ ZNG-1 and $M 15 Z N G-1: T_{\text {eff }}, \log g$ and $\xi$}

Effective temperature ( $\left.T_{\text {eff }}\right)$ estimates for M $10 \mathrm{ZNG}-1$ and M 15 ZNG-1 were made by balancing the silicon ionization equilibrium deduced from the Si III and Si IV ions. Logarithmic surface gravities were deduced from a direct comparison of computed profiles of the Balmer hydrogen lines $\mathrm{H} \epsilon, \mathrm{H} \delta, \mathrm{H} \gamma$ and $\mathrm{H} \beta$ with the observations. Iterating this procedure led to a convergence of the $T_{\text {eff }}$ and $\log g$ values, which are listed in Table 1 . The microturbulence velocity $(\xi)$ of M 10 ZNG-1 was determined by choosing a value that produced a straight line fit with zero gradient for $\mathrm{O} / \mathrm{H}$ abundance vs. O II line equivalent widths (see Keenan \& Lennon 1984; Gies \& Lambert 1992). Few O absorption features were apparent in the spectra of M 15 ZNG-1, and therefore $\xi_{\text {LTE }}$ was deduced by fitting $\mathrm{N} / \mathrm{H}$ abundance vs. $\mathrm{N}$ II line equivalent widths.

\subsubsection{NGC 6712 ZNG-1: $T_{\text {eff }}$, $\log g$ and $\xi$}

Since only Si II lines are observed in the spectra of NGC 6712 ZNG-1, an effective temperature could not be determined from the silicon ionization equilibrium balance. However, many globular cluster post-AGB stars display near solar helium abundances, viz. M 10 ZNG-1 (Mooney et al. 2001), Barnard 29 (Conlon et al. 1994; Moehler et al. 1998b) and ROB 162 in NGC 6397 (Heber \& Kudritzki 1986). As a result, the effective temperature was estimated from the requirement that the helium abundance was solar. The surface gravity was again determined from the comparison of model profiles to observed Balmer hydrogen features, with the $\mathrm{H} \epsilon, \mathrm{H} \delta$ and $\mathrm{H} \gamma$ features being used. The microturbulence was estimated using the Fe II line spectrum and a similar procedure to that described in Sect. 3.2.1. The adopted atmospheric parameters are again summarized in Table 1.

\section{Absolute LTE abundances}

The adopted atmospheric parameters listed in Table 1 were used to derive stellar chemical compositions for the programme stars. Absolute LTE abundances on the logarithmic scale $12+\log (X / \mathrm{H})$ are quoted in Table 3 . For comparison purposes, also listed in Table 3 are the mean abundance values reported by Gies \& Lambert (1992) for a sample of 39 early B-type stars of luminosity classes V-Ib found in the local field. These are non-LTE values for $\mathrm{He}, \mathrm{C}, \mathrm{N}, \mathrm{O}, \mathrm{Si}$ and $\mathrm{Al}$, and LTE for $\mathrm{S}$ and $\mathrm{Fe}$. The non-LTE abundance for $\mathrm{Mg}$ was taken from Kilian (1994) for 21 unevolved B-type stars in the local field and the Ori OB1, Sco-Cen and Sgr OB1 associations. The mean LTE abundances of $\mathrm{Ca}$, $\mathrm{Ti}$ and $\mathrm{Cr}$ are those reported by Venn (1995) for 22 Galactic A-type supergiants.

The absolute abundances summarised in Table 3 show the programme stars to have a metal poor abundance pattern. Furthermore, M 10 ZNG-1 and M 15 ZNG-1 exhibit He abundances which are comparable with the B-type star values reported by Gies \& Lambert (1992). 
Table 2. Adopted atmospheric parameters of the standard stars.

\begin{tabular}{lccc}
\hline \hline Star & $\begin{array}{c}T_{\text {eff }} \\
(\mathrm{K})\end{array}$ & $\begin{array}{c}\log g \\
(\mathrm{dex})\end{array}$ & $\begin{array}{c}\xi \\
\left(\mathrm{km} \mathrm{s}^{-1}\right)\end{array}$ \\
\hline HR 2387 & $28000 \pm 1000$ & $3.8 \pm 0.1$ & 10 \\
HR 6825 & $10500 \pm 1000$ & $1.8 \pm 0.1$ & 12 \\
\hline
\end{tabular}

\subsection{Standard stars: $H R 2387$ \& HR 6825}

In Table 3 absolute abundances are also presented for the two bright, B-type stars HR 2387 and HR 6825, derived using the atmospheric parameters listed in Table 2. These parameters were determined using similar methods to those employed for the programme stars, as discussed in Sect. 3.2. Comparison of their absolute abundances with Population I values demonstrate the Population I chemical compositions of HR 2387 and HR 6825. Indeed, Hambly et al. (1997) has previously identified HR 2387 as having a normal, Population I composition. Likewise, Lee et al. (2004) has used HR 6825 to analyse a late B-type supergiant in the Magellanic Clouds. Furthermore, the absolute abundances derived for HR 6825 are in good agreement with those derived for $\beta$ Ori (三Rigel), a bright, normal B8Ia supergiant (see McErlean et al. 1999).

However, the following caveats should be noted. First, the nitrogen abundance estimate obtained for HR 6825 ( $~ 8.6$ dex) is significantly enhanced relative to normal, B-type dwarfs $(\sim 7.8 \mathrm{dex})$. This is consistent with the evolutionary status of HR 6825, since the photospheres of supergiants are enhanced in nitrogen as a consequence of the CNO bi-cycle. Secondly, the equivalent width estimate for the Ca K $3933.66 \AA$ feature in HR 6825 may be contaminated with an interstellar component. Hence, the calcium abundance estimate derived for this star should be treated with caution. Thirdly, our absolute iron abundance estimates are slightly lower than that found by, for example, Gies \& Lambert (1992) for Population I objects. However, they are entirely consistent with that found for other young, B-type stars analysed using similar modelatmosphere techniques to that adopted here (see Hambly et al. 1997). Furthermore, the use of these standards in the analysis of Magellanic System stars (see Rolleston et al. 2002, 2003) leads to the canonical iron deficiencies of these galaxies reported in the literature.

\section{Differential LTE abundances}

Absolute LTE abundances may contain systematic errors due to uncertainties in atmospheric parameters and atomic data, or simplifications in the model atmosphere analysis (e.g. the assumption of LTE). Additionally, caution must be exercised when interpreting the absolute abundance ratios of the programme stars relative to mean absolute values (see Table 3), since different model-atmosphere codes, spectral features and atomic datasets may have been used in the two sets of analyses. To minimise these errors, we have performed a differential abundance analysis for each of the programme stars, relative to our standard stars. M 10 ZNG-1 and M 15 ZNG-1 were analysed differentally relative to the standard star HR 2387, while NGC 6712 ZNG-1 was examined relative to the standard HR 6825.

A differential analysis is carried out on a line-by-line basis. For any given species, the abundances of absorption features common to to both the programme and comparison star are directly compared. The differential abundance estimates of each observed line are then averaged to give a mean differential abundance for the species concerned. The differential abundances of M 10 ZNG-1, M 15 ZNG-1 and NGC 6712 ZNG-1 are summarised in Table 4 and discussed below.

\section{1. $M 10 Z N G-1$}

Mooney et al. (2001) recently analysed a spectrum of M 10 ZNG-1 obtained using the AAT 3.9-m telescope. Those data were of a lower signal-to-noise ratio and smaller wavelength coverage than the VLT/UVES spectra presented here, which precluded the determination of abundance estimates for key elements $-\mathrm{C}, \mathrm{S}$ and Fe. Generally, for the subset of elements in common (viz. $\mathrm{N}, \mathrm{O}, \mathrm{Mg}$ and $\mathrm{Si}$ ), the new, more accurate, differential abundances derived here (see Table 4) are in reasonable agreement with those found previously. As with many post-AGB objects, M 10 ZNG-1 has a He abundance which is comparable to typical Population I values. For the metals, we derive an $\mathrm{O}$ underabundance of -0.9 dex, whilst $\mathrm{Si}, \mathrm{S}$ and $\mathrm{Mg}$ are all deficient by $\sim 1.0$ dex. A mean $\mathrm{N}$ underabundance of -0.7 dex was determined, showing a slight enhancement relative to the other species.

Given the better quality and higher $S / N$ ratio of the UVES data, it was possible to reliably measure many more absorption features. This in turn should more accurately constrain the chemical composition of M 10 ZNG-1. Indeed, the higher $S / N$ spectra permitted the observation of species with much weaker spectral features not previously observed, notably C (see Fig. 1a), S and Fe. Whereas Mooney et al. (2001) derived only an upper limit for $C$ of $<-1.29$ dex using $\mathrm{C}$ III lines, the new spectra yield an abundance (from C III $4647.42 \AA$ ) of -2.46 dex. Iron was found to be underabundant by approximately -0.8 dex. Summarizing, M 10 ZNG-1 appears to exhibit a mean metal deficiency of approximately -0.9 dex.

\section{2. $M 15 Z N G-1$}

The differential analysis reveals a near normal He abundance with a $\mathrm{N}$ underabundance of -0.9 dex and a mean $\mathrm{Si}$ value of -1.4 dex. Despite an effective temperature of $T_{\text {eff }}=28000 \mathrm{~K}$, only one O absorption line was observed (O II 4649.14 $\AA$ ) leading to a large $\mathrm{O}$ deficiency of $-2.2 \mathrm{dex}$. Also, the $\mathrm{Mg}$ II feature detected in the spectra of M 15 ZNG-1 was extremely weak $(<7 \mathrm{m \AA})$ so the derived differential abundance of $<-1.6 \mathrm{dex}$ is less well constrained and is also an upper limit. Carbon absorption features were not observed in the spectra of M 15 ZNG-1, and hence upper limits were evaluated using the method discussed by Mooney et al. (2001). This led to a minimum underabundance of -2.0 dex. Using the $\alpha$-elements as tracers of 
Table 3. Absolute abundance analysis.

\begin{tabular}{|c|c|c|c|c|c|c|c|c|c|}
\hline \multirow{2}{*}{$\begin{array}{l}\text { Species } \\
\mathrm{He} \mathrm{I}\end{array}$} & \multicolumn{2}{|c|}{ M 10 ZNG-1 } & \multicolumn{2}{|c|}{ M 15 ZNG-1 } & \multirow{2}{*}{$\begin{array}{c}\text { HR } 2387 \\
10.79\end{array}$} & \multicolumn{2}{|c|}{ NGC 6712 ZNG-1 } & \multirow{2}{*}{$\frac{\text { HR } 6825}{10.87}$} & \multirow{2}{*}{$\frac{\text { Pop. } \mathrm{I}^{3}}{11.00}$} \\
\hline & $10.67 \pm 0.19$ & (4) & $10.78 \pm 0.15$ & (5) & & $10.81 \pm 0.10$ & (6) & & \\
\hline C II & & & $<6.83 \pm 0.54$ & (2) & 8.25 & & & 8.15 & 8.20 \\
\hline C III & 5.95 & (1) & $<7.31 \pm 0.11$ & (2) & 8.42 & & & & \\
\hline N II & $7.19 \pm 0.19$ & (22) & $6.91 \pm 0.08$ & (7) & 7.92 & & & 8.61 & 7.81 \\
\hline N III & $7.34 \pm 0.15$ & (2) & & & 8.19 & & & & \\
\hline O II & $7.66 \pm 0.15$ & (69) & 6.32 & (1) & 8.55 & & & & 8.68 \\
\hline Mg II & $6.17 \pm 0.27$ & $(2)$ & $<5.66 \pm 0.18$ & (2) & 7.27 & $6.70 \pm 0.19$ & (4) & 7.38 & 7.38 \\
\hline $\mathrm{Al}$ II & & & & & & 5.43 & (1) & 6.02 & 6.30 \\
\hline Si II & & & & & 6.87 & $6.66 \pm 0.11$ & (6) & 7.29 & \\
\hline Si III & $6.22 \pm 0.12$ & (3) & $5.84 \pm 0.04$ & (3) & 7.38 & & & & 7.58 \\
\hline Si IV & $6.22 \pm 0.12$ & $(2)$ & $5.84 \pm 0.08$ & (2) & 7.38 & & & & 7.58 \\
\hline S III & $5.83 \pm 0.10$ & (3) & & & 7.01 & & & & 7.21 \\
\hline Ca II & 5.01 & (1) & & & & $4.90 \pm 0.42$ & (2) & 6.79 & 6.34 \\
\hline Ti II & & & & & & $3.67 \pm 0.09$ & (2) & 4.97 & 4.86 \\
\hline Cr II & & & & & & 3.64 & (1) & 5.09 & 5.61 \\
\hline Fe II & & & & & & $5.96 \pm 0.16$ & (18) & 7.00 & \\
\hline $\mathrm{Fe}$ III & $6.38 \pm 0.10$ & $(2)$ & & & 7.04 & & & & 7.48 \\
\hline
\end{tabular}

1 Errors (where quoted) refer to the $1 \sigma$ standard deviation of abundance estimates obtained from individual lines. Uncertainties on the single measurements should be typically less than 0.2 dex.

2 The numbers in parentheses after each abundance estimate represent the number of absorption lines used in the model-atmosphere analysis.

3 Normal, Population I B-star values have been taken from Gies \& Lambert (1992) - non-LTE abundances for He, C, N, O, \& Si, and Kilian (1994), viz. non-LTE abundance for Mg and LTE abundances for S \& Fe. Normal, Population I abundances (LTE) of Ca, Ti \& Cr are those reported by Venn (1995) for Galactic A-type supergiants.

metallicity, M 15 ZNG-1 appears to be metal-poor by at least -1.7 dex.

\subsection{NGC 6712 ZNG-1}

The differential analysis (excluding $\mathrm{Ca}$ ) yields a mean metallicity of approximately $-0.8 \mathrm{dex}$ for NGC $6712 \mathrm{ZNG}-1$. There is some evidence for $\mathrm{Si}$ to be slightly overabundant $(-0.5 \mathrm{dex})$ and $\mathrm{Cr}$ slightly underabundant $(-1.2 \mathrm{dex})$ relative to this mean value. The large $\mathrm{Ca}$ underabundance of -2.2 dex may not be real as the equivalent width estimate in the standard star may be contaminated by an interstellar component.

\section{Discussion}

\subsection{Evolutionary status, luminosity, mass and distance}

It is important to establish the evolutionary status of our programme stars, and to confirm that they are indeed associated with the respective globular clusters. Furthermore, we can compare our spectroscopic distance estimates with published globular cluster distances to perform a consistency check on our choice of atmospheric parameters.

The programme stars have been previously identified as being members of their respective globular clusters based on radial velocity measurements and proper motion studies (see Strom et al. 1970 and Chen et al. 2000 for M 10 ZNG-1;
Cudworth 1976 for M 15 ZNG-1; and Cudworth 1988 for NGC 6712 ZNG-1). Radial velocity estimates obtained from our high-resolution spectra are presented in Table 5. These are in excellent agreement with the mean cluster radial velocities quoted by Harris (1996).

Figure 2 shows the evolutionary tracks of Schönberner (1983, 1987) plotted in the $T_{\text {eff- }} \log g$ diagram. By comparing their derived atmospheric parameters (see Table 1), we find the programme stars to be located close to the lowest mass postAGB track, implying a current mass of $\sim 0.55 M_{\odot}$ in all cases. This places M 10 ZNG-1, M 15 ZNG-1 and NGC 6712 ZNG-1 in a region of the $T_{\text {eff- }}-\log g$ diagram where other well known globular cluster post-AGB objects are known to reside, viz. ZNG-1 in M 5 (Napiwotzki \& Heber 1998), Barnard 29 in M 13 (Conlon et al. 1994; Moehler et al. 1998b), ROB 162 in NGC 6397 (Heber \& Kudritzki 1986) and ROA 5701 in $\omega$ Centauri (Moehler et al. 1998b). It is evident from the sample of hot post-AGB stars studied thus far, that most objects are biased towards the lower mass evolutionary tracks. This is most likely a selection effect as lower mass post-AGB stars have longer evolutionary time scales $-\Delta \tau_{\mathrm{PAGB}} \sim 10^{5} \mathrm{yr}$ for $0.546 M_{\odot}$ cf. $\Delta \tau_{\mathrm{PAGB}} \sim 10^{4} \mathrm{yr}$ for $0.565 M_{\odot}$. However, postearly AGB stars also traverse this region of the $T_{\text {eff }}-\log g$ diagram (see Dorman et al. 1993). These are stars which evolve from the extended horizontal branch (EHB) with sufficient envelope mass to ascend the AGB, but which evolve off the AGB prior to the onset of thermal pulsing. Dorman et al. (1993) have related the duration of post-AGB evolution to 
Table 4. Differential abundance analysis.

\begin{tabular}{|c|c|c|c|c|c|c|}
\hline \multirow{3}{*}{$\begin{array}{l}\text { Species } \\
\mathrm{He}_{\mathrm{I}} \\
\mathrm{C}_{\text {II }}\end{array}$} & \multicolumn{2}{|c|}{ M 10 ZNG-1 } & \multicolumn{2}{|c|}{ M 15 ZNG-1 } & \multicolumn{2}{|c|}{ NGC 6712 ZNG-1 } \\
\hline & $-0.22 \pm 0.08$ & (2) & $-0.04 \pm 0.09$ & (2) & $-0.06 \pm 0.17$ & (6) \\
\hline & & & $<-1.07 \pm 0.02$ & (2) & & \\
\hline C III & -2.46 & $(1)$ & $<-1.99 \pm 0.17$ & (2) & & \\
\hline N II & $-0.77 \pm 0.13$ & (10) & $-0.88 \pm 0.09$ & (3) & & \\
\hline N III & $-0.72 \pm 0.12$ & $(2)$ & & & & \\
\hline O II & $-0.87 \pm 0.11$ & (46) & -2.19 & (1) & & \\
\hline $\mathrm{Mg}$ II & $-1.10 \pm 0.27$ & $(2)$ & $<-1.61 \pm 0.18$ & (2) & $-0.84 \pm 0.26$ & (4) \\
\hline $\mathrm{Al}$ II & & & & & -0.59 & (1) \\
\hline Si II & & & & & $-0.46 \pm 0.16$ & (3) \\
\hline Si III & $-1.00 \pm 0.04$ & (2) & $-1.45 \pm 0.09$ & (2) & & \\
\hline Si IV & -1.01 & (1) & -1.38 & (1) & & \\
\hline S III & $-0.93 \pm 0.02$ & (2) & & & & \\
\hline Ca II & & & & & -2.18 & (1) \\
\hline Cr II & & & & & -1.24 & (1) \\
\hline Fe II & & & & & $-0.93 \pm 0.12$ & (18) \\
\hline Fe III & $-0.79 \pm 0.06$ & (2) & & & & \\
\hline
\end{tabular}

${ }^{1}$ Errors (where quoted) refer to the $1 \sigma$ standard deviation of abundance estimates obtained from individual lines. Uncertainties on the single measurements should be typically less than 0.2 dex.

2 The numbers in parentheses after each abundance estimate represent the number of absorption lines used in the model-atmosphere analysis.

Table 5. Radial velocity and spectroscopic distance estimates.

\begin{tabular}{lcccccr}
\hline \hline Star & $\log L / L_{\odot}$ & $\begin{array}{c}v_{\mathrm{lsr}} \\
\left(\mathrm{km} \mathrm{s}^{-1}\right)\end{array}$ & $\begin{array}{c}v_{\mathrm{lsr}}{ }^{-1} \\
\left(\mathrm{~km} \mathrm{~s}^{-1}\right)\end{array}$ & $\begin{array}{c}d \\
(\mathrm{kpc})\end{array}$ & $\begin{array}{c}z \\
(\mathrm{kpc})\end{array}$ & $\begin{array}{c}d^{1} \\
(\mathrm{kpc})\end{array}$ \\
\hline M 10 ZNG-1 & 3.26 & +91.7 & +91.5 & $4.0 \pm 1.0$ & $+1.6 \pm 0.4$ & 4.4 \\
M 15 ZNG-1 & 3.17 & -99.6 & -94.8 & $9.5 \pm 2.4$ & $-4.4 \pm 1.1$ & 10.3 \\
NGC 6712 ZNG-1 & 3.16 & -100.0 & -92.4 & $6.5 \pm 1.6$ & $-0.5 \pm 0.1$ & 6.9 \\
\hline
\end{tabular}

${ }^{1}$ Mean globular cluster values adopted from Harris (1996).

the luminosity at which the star traverses the H-R diagram. They conclude that post-early AGB stars are generally less luminous $\left(\log L / L_{\odot}<3\right.$ ) than true post-AGBs. Hence, given the luminosities quoted in Table 5, we believe M 10 ZNG1, M 15 ZNG-1 and NGC 6712 ZNG-1 to be low mass postAGB stars.

Spectroscopic distance estimates were determined by first calculating absolute visual magnitudes from the stellar masses, atmospheric parameters and the bolometric corrections of Kurucz (1979). Estimates of the foreground reddening towards M 10, M 15 and NGC 6712 were obtained from Harris (1996), which combined with the apparent visual magnitudes of the programme stars given by de Boer (1985), led to the distance estimates given in Table 5. These are in good agreement with the distance to the respective globular clusters quoted by Harris (1996); hence, re-affirming our choice of atmospheric parameters for the programme stars.

\subsection{Previous post-AGB analyses}

Recent abundance analyses of both hot and cool post-AGB stars provides a rather complex picture of this late stage of stellar evolution (see van Winckel et al. 1998 for a recent review). In summary, van Winckel (1997) classified the optically bright, F-type PAGBs into three groups, viz.

- C-rich, overabundant in s-process elements and, in some cases a suprasolar He abundance;

- underabundant in Fe by several orders of magnitude, but with approximately Solar values for $\mathrm{CNO}$ and S;

- a mixture of CNO-cycled material and He-burning products with no evidence for an enhancement of the $s$-process elements.

The first class of objects provide clear evidence for an efficent third dredge-up episode, whereas the abundance pattern of the second class of post-AGB stars is interpreted in terms of a gasdust separation event having occurred. The remaining objects display a general trend that is indicative of an old population.

Early studies of high-latitude, B-type, post-AGB candidates revealed abundance patterns that were characterized by a large C deficiency (see McCausland et al. 1992); a similar result was also obtained by Conlon et al. (1994) for the globular cluster star Barnard 29. However, in many aspects, such objects are not dissimilar to the third group of post-AGB objects discussed by van Winckel (1997). For example, Barnard 29 and M 10 ZNG-1 exhibit N enhancements relative to the 


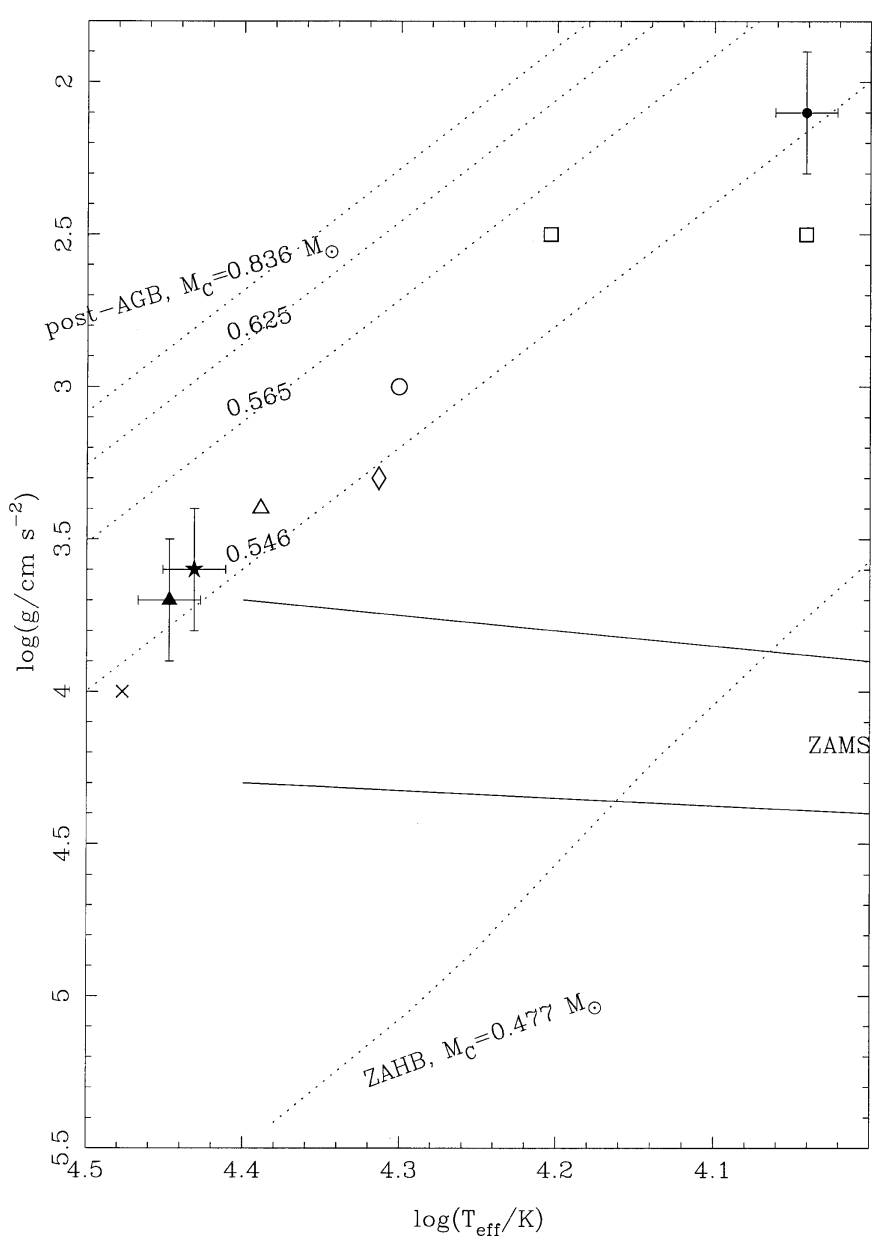

Fig. 2. Position of M 10 ZNG-1 (filled star), M 15 ZNG-1 (filled triangle) and NGC 6712 ZNG-1 (filled circle) in the $\log T_{\text {eff }}-\log g$ diagram, showing the post-AGB evolutionary tracks of Schönberner $(1983,1987)$ for masses of 0.546 and $0.565 M_{\odot}$, and those of Blöcker (1995) for 0.625 and $0.836 M_{\odot}$. Also shown are the positions of other globular cluster post-AGB stars; Conlon et al. (1994) (open circle), Moehler et al. (1998b) (open triangle), Moehler et al. (1998a) (open diamond), Dixon et al. (1994, 1995) (open squares) and Landsman et al. (1992) (cross).

other metals. Table 6 of Ryans et al. (2003) illustrated the remarkable agreement between the abundance pattern of this latter object and the optically bright, F-type, post-AGB star HD 107369 which shows evidence of a C deficiency. Indeed, van Winckel (1997) had previously commented that the observational evidence points to a different evolutionary history between the dusty, high-latitude, F-type post-AGBs and these low-mass B-type post-AGBs, but that the latter may evolve from objects such as HD 107369.

More recently, Ryans et al. (2003) analysed two relatively high mass, B-type, post-AGB stars (viz. LSIV -12 111 and HD 341617) using non-LTE model-atmosphere techniques. Their photospheres display an approximately uniform underabundance of $0.4 \mathrm{dex}$, similar to those observed for the optically, bright F-type PAGB stars SAO 239853 and SAO 173329. Ryans et al. concluded that these high mass, B-type post-AGBs with circumstellar material may be the hotter analogues of the third group of F-type post-AGBs discussed by van Winckel (1998); hence, indicating a common origin for such objects.

\subsection{Comparison with other stellar abundance patterns}

Given these studies, we have attempted to compare the results obtained for our three globular cluster stars with the studies of the cool PAGBs and globular cluster giants. However, PAGB/Population II abundances are generally compared with Solar values and are given relative to Fe. Unfortunately, it was not possible to derive an $\mathrm{Fe}$ abundance estimate for M 15 ZNG-1; additionally, significant differences exist between the absolute abundances derived for the Sun and normal, Population I B-type stars in the Solar neighnourhood (see Gies $\&$ Lambert 1992). Thus, we believe our differential abundances presented in Table 4 to be more reliable and have adopted the approach taken by Ryans et al. (2003). It is not possible to obtain "true" differential abundances of globular cluster giants relative to normal, Population I objects of similar atmospheric parameters. Therefore, in Table 6 the abundances presented for the globular cluster giants are simply those obtained from the listed sources, measured relative to $\mathrm{H}$ and compared with the Sun. Furthermore, we have included in Table 6 results for Barnard 29 and the optically bright, F-type post-AGB star HD 107369 for comparison.

\subsubsection{10 ZNG-1}

M 10 ZNG-1 displays an abundance pattern similar to that derived previously for Barnard 29 in M 13 (see Table 6). The relative contributions of $\mathrm{CNO}$ in the photosphere of $\mathrm{M} 10 \mathrm{ZNG}-1$, viz. $\Delta[\mathrm{C} / \mathrm{O}] \sim-1.6 \mathrm{dex}$ and $\Delta[\mathrm{N} / \mathrm{O}] \sim+0.2 \mathrm{dex}$, are indicative of $\mathrm{H}$ burning products having been convected to the stellar surface. This is believed to occur during the first and second dredge up episodes on the AGB. Given that the abundance pattern is inconsistent with the products of He burning, Conlon et al. (1994) had concluded that such stars must evolve off the AGB before the onset of the third dredge-up. Inspection of Table 6 affirms the assertion of Ryans et al. (2003) that M 10 ZNG-1 and the optically bright post-AGB star HD 107369 possess similar abundance patterns. Iron is observed to have a near normal abundance relative to the $\alpha$-elements; yielding an abundance ratio of $\Delta[\alpha / \mathrm{Fe}] \sim+0.15 \mathrm{dex}$. This is entirely consistent with the third class of cool post-AGB stars studied by van Winckel (1998) and in particular the abundance ratio of $\Delta[\alpha / \mathrm{Fe}] \sim-0.1$ dex found for HD 107369.

\subsection{2. $\mathrm{M} 15 \mathrm{ZNG}-1$}

Unfortunately, the faintness of M 15 ZNG-1 $(V \sim 15)$ and the low metallicity of this globular cluster $([\mathrm{Fe} / \mathrm{H}]=-2.15$, Zinn 1985) precluded the measurement of abundances for several key elements, viz. $\mathrm{C}, \mathrm{Mg}$ and Fe. Thus, it is difficult to comment on the precise evolutionary history of this object. Furthermore, given the discussion in Sect. 6.4.2 concerning the discrepancy between the Fe abundance determinations of M 10 ZNG-1 and M 10 giants, we do not believe 
Table 6. Comparison of globular cluster post-AGBs and giants.

\begin{tabular}{lcccccccc}
\hline \hline Element & M 10 & M 15 & NGC 6712 & M 10 & M 15 & NGC 6712 & M 13 & HD 107369 \\
& ZNG- $^{1}$ & ZNG- $^{1}$ & ZNG- $^{1}$ & Giants & Giants & Giants & Barnard 297 & \\
\hline $\mathrm{C}$ & -2.5 & $<-2.0$ & & $-2.7^{2}$ & $-3.6^{4}$ & & $<-1.8$ & -1.3 \\
$\mathrm{~N}$ & -0.7 & -0.9 & & $-0.5^{2}$ & $-1.3^{4}$ & & -0.8 & -0.7 \\
$\mathrm{O}$ & -0.9 & -2.2 & & $-1.5^{3}$ & $-2.3^{5}$ & & -1.2 & -1.1 \\
$\mathrm{Mg}$ & -1.1 & $<-1.6$ & -0.8 & & $-2.0^{5}$ & & -1.5 & -0.9 \\
$\mathrm{Si}$ & -1.0 & -1.4 & -0.5 & $-1.2^{3}$ & $-1.8^{5}$ & & -1.4 & -0.9 \\
$\mathrm{~S}$ & -0.9 & & & & & & $<-0.8$ & -1.0 \\
$\mathrm{Fe}$ & -0.8 & & -0.9 & $-1.5^{3}$ & $-2.4^{5}$ & $-0.8^{6}$ & & -1.1 \\
\hline
\end{tabular}

1 This work.

2 Briley \& Smith (1993).

3 Kraft et al. (1995).

4 Trefzger et al. (1993).

5 Sneden et al. (1997).

6 Paltrinieri et al. (2001).

7 Conlon et al. (1994).

8 van Winckel (1997).

that it is appropriate to adopt a value for the Fe abundance in our comparisons. However, the data yield an abundance ratio of $\Delta[\mathrm{N} / \mathrm{O}] \sim+1.3 \mathrm{dex}$, which is indicative of $\mathrm{H}$ burning products having been mixed with the stellar photosphere. The $\alpha$-elements $\mathrm{O}$ and $\mathrm{Si}$ give significantly different deficiencies with $\Delta[\mathrm{Si} / \mathrm{O}] \sim+0.8 \mathrm{dex}$. This ratio can not be explained alone by depletion of the initial photospheric oxygen abundance due to the ON-cycle. Rather, differences are more likely to have existed in the metallicity of the progenitor gas. Indeed, comparison of the $\mathrm{Si} / \mathrm{O}$ abundance in M 15 ZNG-1 with M 15 giants supports such a conclusion as the latter objects yield $\triangle[\mathrm{Si} / \mathrm{O}] \sim+0.5$ dex (see Table 6).

\subsubsection{NGC 6712 ZNG-1}

NGC 6712 ZNG-1, being much cooler than M 10 ZNG-1 and M 15 ZNG-1 (see Table 1), shows no C, N or O absorption features. Thus, it is not possible to infer if this star evolved off the AGB before the third dredge-up. However, constraints on the evolutionary history of this object can be made by comparing elements from sites of different nucleosynthetic origin, viz, the $\alpha$-elements $(\mathrm{Mg}, \mathrm{Si}, \mathrm{Ca})$ and iron-group elements $(\mathrm{Cr}$, $\mathrm{Fe})$. Excluding $\mathrm{Ca}$, an abundance ratio of $\Delta[\alpha / \mathrm{Fe}] \sim+0.25 \mathrm{dex}$ was obtained. Calcium is deficient by 1.3 dex relative to $\mathrm{Fe}$, which may be interpreted as evidence for a gas-dust fractionation event having occurred. Indeed, McCausland et al. (1992) reported similar large underabundances of $\mathrm{Ca}$ for the two field post-AGB stars PHL 174 and LSIV -401. However, the comments given in Sect. 5.3 should be borne in mind whenever interpreting the result for $\mathrm{Ca}$. Furthermore, no such depletion is observed for other refractory elements such as $\mathrm{Si}$.

\subsection{Comparison with cluster giants}

The chemical compositions of the programme stars are compared with the respective cluster giants in Table 6; references to the data sources are provided as footnotes.

\subsubsection{15 and NGC 6712}

Unfortunately, NGC 6712 has not been the subject of intensive abundance investigations. Indeed, it was only possible to compare results presented in this paper with a recent determination of the cluster Fe abundance by Paltrinieri et al. (2001). These values are in excellent agreement. Our comparisons for M 15 ZNG-1 are limited by the lack of data for C, Mg, S and Fe (see Sect. 6.3.2). However, in general our abundance estimates and upper limits for $\mathrm{C}$ and $\mathrm{Mg}$ are compatible with that derived for M 15 giants.

\subsection{2. $\mathrm{M} 10$}

Differential abundance estimates of $\mathrm{C}, \mathrm{N}$ and $\mathrm{Si}$ for M 10 ZNG-1 are in good agreement with that obtained for M 10 cluster giants (see Table 6). This provides further evidence that M 10 ZNG-1 evolved of the AGB before the third dredge-up episode occurred. However, significant discrepancies exist between $\mathrm{M} 10 \mathrm{ZNG}-1$ and the giants for $\mathrm{O}$ and Fe. Although the ratio $\Delta[\mathrm{O} / \mathrm{Fe}]$ is approximately 0.0 dex for both, our determination of the Fe abundance in M 10 ZNG-1 is certainly not consistent with the cluster metallicity of $\Delta[\mathrm{Fe} / \mathrm{H}] \sim-1.5 \mathrm{dex}$ (Kraft et al. 1995).

It is worthwhile re-iterating that we believe our Fe abundance estimate to be secure for M 10 ZNG-1, given that the observational data is of high signal-to-noise ratio and a careful differential model-atmosphere analysis has been performed (see Sects. 4.1, 5.1). Unfortunately, the number of investigations for which $\mathrm{Fe}$ abundances have been determined for hot, globular cluster post-AGB stars are small. Excluding this work, Moehler et al. (1998b) reported a large Fe underabundance for Barnard 29 in M 13 - approximately 0.5 dex below the nominal cluster abundance. A similar result was found by Dixon \& Hurwitz (1998) for this star. Furthermore, Moehler et al. derived an $\mathrm{Fe}$ abundance for ROA 5701 in $\omega$ Centauri that was lower than previously observed for any star in the globular 
cluster. However, these studies compared absolute abundances deduced for hot, B-type, post-AGB stars with absolute solar abundances. Indeed, it has been recognized that the consequence of not comparing "like with like" can be the introduction of systematic errors in the analysis (see Pagel 1992).

Assuming that our model-atmosphere analysis is secure, it is important to consider evolutionary processes that might lead to the increase in the $\mathrm{Fe}$ content in the photosphere of M 10 ZNG-1. Gas-dust fractionation events (which are thought to occur in the extended atmospheres of cool post-AGB stars; see van Winckel 1998), cannot explain the observed abundance pattern of M 10 ZNG-1 as this would lead to an Fe underabundance. Indeed, Napiwotzki et al. (2001) explained the low Fe abundance of the halo, hot, post-AGB star BD $+33^{\circ} 2642$ in terms of such an event having occurred.

Both field and globular cluster blue Horizontal Branch (BHB) stars are known to display peculiar abundance patterns, with elements such as $\mathrm{Si}, \mathrm{P}$ and $\mathrm{Fe}$ being enhanced relative to He, C and N (see, for example, Galspey et al. 1989; Castelli et al. 1997; Hambly et al. 1997). Michaud et al. (1983) predicted that in the stable, non-convective atmospheres of hotter, higher gravity HB stars which permit the gravitational settling of $\mathrm{He}$, the levitation of metals with larger radiative cross sections will also occur. Indeed, Moehler et al. $(1999,2000)$ derived $\mathrm{Fe}$ abundances for $19 \mathrm{HB}$ stars in NGC 6752; these were enriched by a factor of 50 relative to the cluster abundance. More recently, Behr et al. (1999, 2000a, 2000b) concluded that circulation currents, induced by higher rotational velocities, prohibited the radiative levitation of metals in HB stars with $v \sin i \geq 10 \mathrm{~km} \mathrm{~s}^{-1}$.

M $10 \mathrm{ZNG}-1$ is on the post-AGB phase of stellar evolution. Thus, any photospheric abundance signature resulting from diffusive processes on the horizontal branch should have been removed by convection on the AGB. However, in Sect. 6.3.1 we concluded that this star appears to have evolved off the AGB before this final convective process occurred, as the products of He burning are not manifested in the abundance pattern. Rather, the photosphere has a large $\mathrm{C}$ underabundance (together with a $\mathrm{N}$ enhancement) - evidence for the convection of products of $\mathrm{H}$ burning to the stellar surface. Furthermore, our $\mathrm{Mg}$ abundance estimate of $\Delta[\mathrm{Mg} / \mathrm{H}]=-1.1 \pm 0.3 \mathrm{dex}$ is also enhanced relative to the accepted cluster metallicity of approximately $-1.5 \mathrm{dex}$ (see Table 7), although we note that the error bar on the former is quite large. However, the derived value is inconsistent with the analyses of Behr et al. (1999, 2000a,b) that found $\mathrm{Mg}$ abundances in blue HB stars to be comparable to the respective cluster metallicities, suggesting that $\mathrm{Mg}$ is apparently immune to levitation processes. Hence, the conundrum remains. We do not believe that the overabundance of $\mathrm{Fe}$ in the photosphere of M $10 \mathrm{ZNG}-1$ relative to the accepted metallicity of M 10 to be due to the signature of radiative levitation processes on the horizontal branch, as no accompanying deficiency of helium persists (see Hambly et al. 1997). Lastly, we have undertaken a literature search of recent metallicity determinations for M 10; these are presented in Table 7. This list is not exhaustive; rather, it is intended to be a representative selection of that which may be found in the literature. Table 7 yields a mean metallicity of $\Delta[\mathrm{Fe} / \mathrm{H}]=-1.46 \pm 0.10 \mathrm{dex}$; although,
Table 7. Previous metallicity determinations for M 10.

\begin{tabular}{lc}
\hline \hline Reference & {$[\mathrm{Fe} / \mathrm{H}]$} \\
\hline Pilachowski et al. (1983) & -1.32 \\
Smith (1984) & -1.34 \\
Pilachowski (1984) & -1.39 \\
Zinn \& West (1984) & -1.60 \\
Hesser \& Shawl (1985) & -1.56 \\
Brodie \& Hanes (1986) & -1.46 \\
Hurley et al. (1989) & -1.54 \\
Kraft et al. (1995) & -1.52 \\
Carretta \& Gratton (1997) & -1.41 \\
\hline
\end{tabular}

the error associated with any one abundance determination is likely to be much larger. For example, Hurley et al. (1989) suggest an uncertainty of \pm 0.35 dex in their estimate. In our comparisons in Table 6, we have adopted the Fe abundance of Kraft et al. (1995) derived from Fe I/Fe II lines in the spectra of $14 \mathrm{cool}$, red giants in M 10. This study yields a Fe abundance of $-1.52 \mathrm{dex}$; furthermore, the highest measured stellar $\mathrm{Fe}$ abundance of $\Delta[\mathrm{Fe} / \mathrm{H}]=-1.36 \mathrm{dex}$ is still much lower than that derived for M $10 \mathrm{ZNG}-1$. Determination of effective temperatures for such cool giants relies on the transformation of photometric colours $(B-V)$ or $(V-K)$ using an empirical temperature calibration. The assignment of the correct effective temperature depends on the adopted value of interstellar reddening. However, Kraft et al. concluded that their abundance determinations were not affected significantly should a higher value of interstellar reddening have been adopted.

Hence, it is difficult to reconcile the Fe abundance estimates derived for M $10 \mathrm{ZNG}-1$ and cluster giants. Clearly, abundance determinations of further hot, post-AGB stars in M 10 and other globular clusters will advance our understanding of this late stage in stellar evolution and may resolve this apparent discrepancy between M 10 Fe determinations.

Acknowledgements. C.J.M. would like to thank the Department of Employment and Learning (DEL) for Northern Ireland and the Isaac Newton Group (ING), La Palma for the award of a CAST research studentship. W.R.J.R. and J.V.S. acknowledge financial assistance from the UK Particle Physics and Astronomy Research Council (PPARC). FPK is grateful to AWE Aldermaston for the award of a William Penney Fellowship. We would also like to thank the support staff at the KECK I and VLT Telescopes for assisting with the observations.

\section{References}

Aller, L. H. 1990, PASP, 102, 1097

Aller, L. H. 1994, ApJ, 432, 427

Aller, L. H., Hyung, S., \& Feibelman, W. A. 1999, Proc. Nat. Acad. Sci. USA, 96, 5366

Behr, B. B., Cohen, J. G., McCarthy, J. K., \& Djorgovski, S. G. 1999, ApJ, 517, L135

Behr, B. B., Cohen, J. G., \& McCarthy, J. K. 2000a, ApJ, 531, L37

Behr, B. B., Djorgovski, S. G., Cohen, J. G., et al. 2000b, ApJ, 528, 849 
Blöcker, T. 1995, A\&A, 299, 755

Briley, M. M., \& Smith, G. H. 1993, PASP Conf. Ser., 48, 184

Brodie, J. P., \& Hanes, D. A. 1986, ApJ, 300, 258

Carretta, E., \& Gratton, R. G. 1997, A\&AS, 121, 95

Castelli, F., Parthasarathy, M., \& Hack, M. 1997, A\&A, 321, 254

Chen, L., Geffert, M., Wang, J. J., Reif, K., \& Braun, J. M. 2000, A\&AS, 145, 223

Conlon, E. S., Dufton, P. L., Keenan, F. P., \& McCausland, R. J. H. 1991, MNRAS, 248, 820

Conlon, E. S., Dufton, P. L., McCausland, R. J. H., \& Keenan, F. P. 1993, ApJ, 408, 593

Conlon, E. S., Dufton, P. L., \& Keenan, F. P. 1994, A\&A, 290, 897

Cudworth, K. M. 1976, AJ, 81, 519

Cudworth, K. M. 1988, AJ, 96, 105

de Boer, K. S. 1985, A\&A, 142, 321

de Boer, K. S. 1987, in 2nd Conference on Faint Blue Stars (Schenectady: L. Davis Press), 95

Dixon, W. V., \& Hurwitz, M. 1998, ApJ, 500, L29

Dixon, W. V., Davidsen, A. F., \& Ferguson, H. C. 1994, AJ, 107, 1388

Dixon, W. V., Davidsen, A. F., \& Ferguson, H. C. 1995, ApJ, 454, 47

Dorman, B., Rood, R. T., \& O'Connell, R. W. 1993, ApJ, 419, 596

Galspey, J. W., Michaud, G., Moffat, A. F. J., \& Demers, S. 1989, ApJ, 339,926

Gies, D. R., \& Lambert, D. L. 1992, ApJ 387, 673

Hambly, N. C., Keenan, F. P., Dufton, P. L., \& Lumsden, S. L. 1996, MNRAS, 278, 811

Hambly, N. C., Rolleston, W. R. J., Keenan, F. P., Dufton, P. L., \& Saffer, R. A. 1997, ApJS, 111, 419

Harris, W. E. 1996, AJ, 112, 1487

Heber, U., \& Kudritzki, R. P. 1986, A\&A, 169, 244

Hesser, J. E., \& Shawl, S. J. 1985, PASP, 97, 465

Howarth, I. D., Murray, J., \& Mills, D. 1994, DIPSO-A Friendly Spectrum Analysis Program, Starlink User Note No. 50.15

Hubeny, I. 1988, Comp. Phys. Comm., 52, 103

Hubeny, I., \& Lanz, T. 1992, A\&A, 262, 501

Hubeny, I., \& Lanz, T. 1995, ApJ, 439, 875

Hurley, D. J. C., Richer, H. B., \& Fahlman, G. G. 1989, AJ, 98, 2124

Keenan, F. P. 1992, QJRAS, 33, 325

Keenan, F. P., \& Lennon, D. J. 1984, A\&A, 130, 179

Kilian, J. 1994, A\&A, 282, 867

Kraft, R. P., Sneden, S., Langer, G. E., Shetrone, M. D., \& Bolte, M. 1995, AJ, 109, 2586

Kurucz, R. L. 1979, ApJS, 40, 1

Landsman, W. B., O'Connell, R. W., Whitney, J. H., et al. 1992, ApJ, 395, L21

Lee, J. K., Dufton, P. L., Rolleston, W. R. J., Lennon, D. J., \& Venn, K. A. 2004, A\&A, in preparation
McCausland, R. J. H., Conlon, E. S., Dufton, P. L., \& Keenan, F. P. 1992, ApJ, 394, 298

McErlean, N. D., Lennon, D. J., \& Dufton, P. L. 1999, A\&A, 349, 553

Michaud, G., Vauclair, G., \& Vauclair, S. 1983, ApJ, 267, 256

Moehler, S., Landsman, W., \& Napiwotzki, R. 1998a, A\&A, 335, 510

Moehler, S., Heber, U., Lemke, M., \& Napiwotzki, R. 1998b, A\&A, 339, 537

Moehler, S., Sweigart, A. V., Landsman, W. B., Heber, U., \& Catelan, M. 1999, A\&A, 346, L1

Moehler, S., Sweigart, A. V., Landsman, W. B., \& Heber, U. 2000, A\&A, 360, 120

Mooney, C. J., Rolleston, W. R. J., Keenan, F. P., et al. 2001, MNRAS, 326, 1101

Mowlavi, N. 1999, A\&A, 344, 617

Napiwotzki, R., \& Heber, U. 1998, in 3rd Conference on Faint Blue Stars, Schenectady, 439

Napiwotzki, R., Herrmann, M., Heber, U., \& Altmann, M. 2001, in a workshop on: Post-AGB objects (proto-planetary nebulae) as a phase of stellar evolution

Pagel, B. E. J. 1992, in New Aspects of Magellanic Cloud Research, ed. G. Klare (Springer), 330

Paltrinieri, B., Ferraro, F. R., Paresce, F., \& De Marchi, G. 2001, AJ, 121,3114

Pilachowski, C. A. 1984, ApJ, 281, 614

Pilachowski, C. A., Sneder, C., \& Wallerstein, G. 1983, ApJS, 52, 241

Rolleston, W. R. J., Trundle, C., \& Dufton, P. L. 2002, A\&A, 396, 53

Rolleston, W. R. J., Venn, K., Tolstoy, E., \& Dufton, P. L. 2003, A\&A, 400, 21

Ryans, R. S. I., Dufton, P. L., Mooney, C. J., et al. 2003, A\&A, 401, 1119

Schönberner, D. 1983, ApJ, 272, 708

Schönberner, D. 1987, in Late Stages of Stellar Evolution, ed. S. Kwok, \& S. R. Pottasch (Dordrecht: Reidel), 359

Schönberner, D., \& Blöcker, T. 1996, Ap\&SS, 245, 201

Smith, H. A. 1984, ApJ, 281, 148

Sneden, C., Kraft, R. P., Shetrone, M. D., et al. 1997, AJ, 114, 1964

Strom, S. E., Strom, K. M., Rood, R. T., \& Iben, I. Jr. 1970, A\&A, 8, 243

Trefzger, C. F., Langer, G. E., Carbon, D. F., Suntzeff, N. B., \& Kraft, R. P. 1983, ApJ, 266, 144

van Winckel, H. 1997, A\&A, 319, 561

van Winckel, H., Decin, L., \& Waelkens, C. 1998, IAUS, 189, 176

Venn, K. A. 1995, ApJS, 99, 659

Zinn, R. 1985, ApJ, 293, 424

Zinn, R., \& West, M. J. 1984, ApJS, 55, 45 